\title{
Predictive value of neutrophil to lymphocyte and platelet to lymphocyte ratio in COVID-19
}

\author{
Shiping Zhu', Lei Dong ${ }^{2}$ and Wanru Cai ${ }^{2^{*}}$ (D)
}

Keywords: Neutrophil to leucocyte ratio, Platelet to lymphocyte ratio, COVID-19

To the Editor,

In a recent study, Dr. Ma [1] investigated the neutrophil to lymphocyte ratio (NLR) in predicting moderate-severe acute respiratory distress syndrome (ARDS) in patients with COVID-19 infection. They reported that NLR was significantly higher ARDS group and was a good predictor for moderate-severe ARDS, with area under curve (AUC) 0.749. This study is well designed. However, several limitations should be mentioned. First, uncontrolled inflammatory response plays a vital role in COVID-19 disease, and both NLR and platelet to lymphocyte ratio (PLR) have been recognized as inflammatory factors in various lung diseases [2,3], such as lung cancer and obstructive lung disease. However, one common limitation is that in most previous studies, NLR or PLR was included in the generalized linear models as a continuous variable, with the assumption that there was a linear association between NLR/PLR and the dependent outcomes. Even in some prediction model [4], researchers only focused on the impact of higher NLR/PLR value while the effect of low NLR/PLR was ignored. However, in most cases, extremely low NLR or PLR represented low neutrophil and platelet, which, according to clinical experience, was also

This comment refers to the article available at https://doi.org/10.1186/ s13054-020-03007-0.

* Correspondence: caiwanru1999@163.com

${ }^{2}$ Respiratory Department, The Second Affiliated Hospital of Zhejiang Chinese Medicine University, No. 318, Chaowang Road, Hangzhou 310000, China Full list of author information is available at the end of the article associated with poor outcomes. Thus, the association between NLR/PLR and outcomes should be nonlinear, and simply including these parameters into a linear model may lead to a biased conclusion. For instance, we estimated an unadjusted association between NLR/PLR and mortality in critically ill patients with lung infection, using data from the MIMIC database (Fig. 1). Although the cohort is different from the current study, the non-linear association, to a certain degree, supports our hypothesis. If this is the case, the predictive value of NLR/PLR in AUC may also be compromised as both high and low NLR/PLR was associated with increased mortality. The following method may improve this limitation: (1) applying non-linear spline regression using the cut-off value of NLR/PLR in logistic model, (2) generating predictive values of the logistic model, and (3) calculating the AUC of the predictive value in predicting mortality. Finally, we thank Dr. Ma et al. for their valuable research.

\section{Abbreviations \\ NLR: Neutrophil to lymphocyte ratio; ARDS: Acute respiratory distress syndrome; AUC: Area under curve; PLR: Platelet to lymphocyte ratio}

\section{Acknowledgements}

None.

\section{Authors' contributions}

WC and LD raised the question and wrote the draft. SZ was responsible for the revision. All authors contributed to the manuscript and approved the final version to be considered for publication.

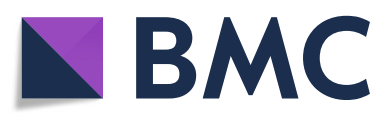

(c) The Author(s). 2020 Open Access This article is licensed under a Creative Commons Attribution 4.0 International License, which permits use, sharing, adaptation, distribution and reproduction in any medium or format, as long as you give appropriate credit to the original author(s) and the source, provide a link to the Creative Commons licence, and indicate if changes were made. The images or other third party material in this article are included in the article's Creative Commons licence, unless indicated otherwise in a credit line to the material. If material is not included in the article's Creative Commons licence and your intended use is not permitted by statutory regulation or exceeds the permitted use, you will need to obtain permission directly from the copyright holder. To view a copy of this licence, visit http://creativecommons.org/licenses/by/4.0/. The Creative Commons Public Domain Dedication waiver (http://creativecommons.org/publicdomain/zero/1.0/) applies to the data made available in this article, unless otherwise stated in a credit line to the data. 


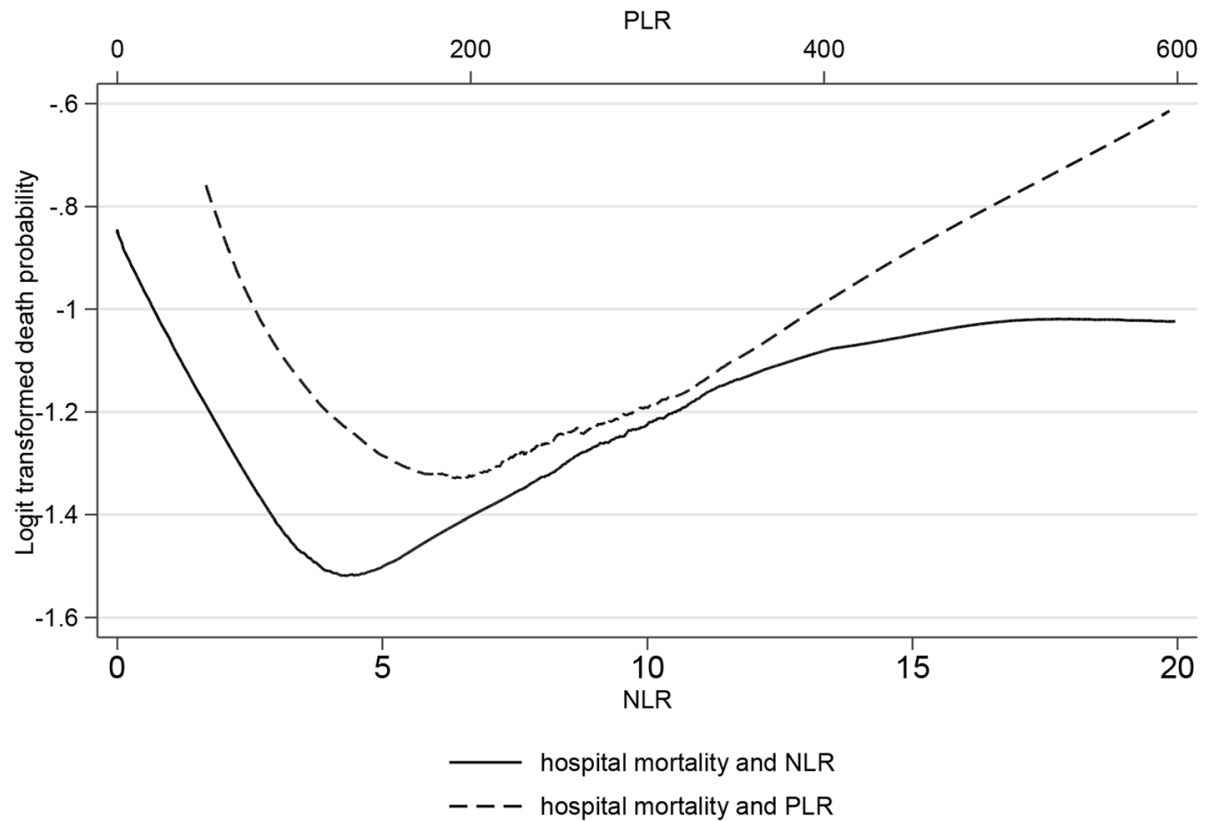

Fig. 1 Non-linear association between NLR/PLR and in-hospital death in patients with lung infection. Abbreviations: NLR neutrophil to lymphocyte, PLR platelet to lymphocyte

\section{Funding}

WC received funding from the National Natural Science Foundation of China (General Program, 81774220) and another tradition Chinese medicine research funding (ID 2012ZGG002) in this research.

\section{Availability of data and materials}

Not applicable.

\section{Ethics approval and consent to participate}

Not applicable.

\section{Consent for publication}

Not applicable.

\section{Competing interests}

The authors declare that they have no competing interests.

\section{Author details}

${ }^{1}$ Respiratory Department, Hangzhou Hospital of Traditional Chinese

Medicine, No. 453, Tiyuchang Road, Hangzhou, Zhejiang, China. ${ }^{2}$ Respiratory Department, The Second Affiliated Hospital of Zhejiang Chinese Medicine University, No. 318, Chaowang Road, Hangzhou 310000, China.

Received: 27 July 2020 Accepted: 17 August 2020

Published online: 28 August 2020

\section{References}

1. Ma A, Cheng J, Yang J, Dong M, Liao X, Kang Y. Neutrophil-to-lymphocyte ratio as a predictive biomarker for moderate-severe ARDS in severe COVID19 patients. Crit Care. 2020;24(1):288.

2. Wang YQ, Zhi QJ, Wang XY, Yue DS, Li K, Jiang RC. Prognostic value of combined platelet, fibrinogen, neutrophil to lymphocyte ratio and platelet to lymphocyte ratio in patients with lung adenosquamous cancer. Oncol Lett. 2017:14(4):4331-8.

3. Liu J, Liu J, Zou Y: Relationship between neutrophil-lymphocyte ratio and short-term prognosis in the chronic obstructive pulmonary patients with acute exacerbation. Biosci Rep. 2019;39(5):BSR20190675.

4. Zhang S, Guo M, Duan L, Wu F, Hu G, Wang Z, Huang Q, Liao T, Xu J, Ma Y, et al. Development and validation of a risk factor-based system to predict short-term survival in adult hospitalized patients with COVID-19: a multicenter, retrospective, cohort study. Crit Care. 2020;24(1):438.

\section{Publisher's Note}

Springer Nature remains neutral with regard to jurisdictional claims in published maps and institutional affiliations. 